\title{
The Function and Role of Rural Revitalization in the Development of Urban Agglomeration
}

\author{
Fengxiang Jiang ${ }^{1}$, Changjun $\mathrm{Du}^{2}$, Runting Xiao ${ }^{3}$ \\ ${ }^{1,2,3}$ Xi'an Peihua University, Xi'an Shaanxi, 710125
}

Keywords: rural revitalization; urban agglomeration; function; role

\begin{abstract}
The 19th CPC National Congress report clearly puts forward the strategy of rural revitalization. With the development of rural development strategy, how to coordinate the development of urban agglomeration and rural areas and promote the development of urban agglomeration by using rural revitalization strategy is worth studying. This paper mainly studies the function and role of rural revitalization in the development of urban agglomeration, to realize the integration of rural development and urban agglomeration development through the effect of the implementation of rural revitalization strategy.
\end{abstract}

\section{Introduction}

As the rural revitalization strategy is proposed, the literature on rural revitalization strategy, urbanization development and urban agglomeration development gradually increases. Xin Zhang (2018) pointed out that there are big differences in the level of economic development, income level, infrastructure, public services and other aspects in urban and rural development to promote rural revitalization through urbanization. According to Yongzheng Kang and Kening Xue(2018), in the context of the rural revitalization strategy, the relationship between rural modernization and urbanization is no longer the mutual relationship in the past, but presents some new features and forms a new relationship of mutual promotion and mutual promotion. Jiming Cai(2018) proposed that the independent implementation of the rural revitalization strategy without the new urbanization strategy would have little effect. The issue of agriculture, rural areas and farmers has always been the top priority of the work of the whole party. The existing literature focuses on the relationship between rural areas and urbanization or the path of urbanization development under the background of rural revitalization strategy; there is little literature on the function and role of rural revitalization in urban agglomeration development

\section{On the rural revitalization strategy}

The rural revitalization strategy is a strategy proposed by comrade Jinping $\mathrm{Xi}$ in the 19th CPC national congress report on Oct 18, 2017. The 2018 central No. 1 document, namely the opinions of the CPC central committee and the state council on the implementation of the rural revitalization strategy, was released on Feb 4, 2018. According to the strategic arrangement of building a moderately prosperous society in all respects and realizing the second centenary goal in two stages proposed by the 19th CPC national congress, the goals and tasks of implementing the rural revitalization strategy are clearly defined. According to the rural revitalization strategy, the level of rural economic development will be improved and the industrial structure will be optimized. The modern agricultural system was built, and the agricultural production efficiency was greatly improved. The quality and ability of farmers can be effectively improved and their income will be increased.

\section{Theoretical and Practical Development of Urban Agglomerations}

\subsection{Concept of Urban Agglomeration}

Urban agglomerations are also known as urban agglomerations, urban belts, urban clusters and 
urban belts. In foreign countries, there is not a completely unified concept corresponding to "urban agglomeration", and the concept of urban agglomeration has Chinese characteristics. The French scholar Guttmann first proposed the concept of megalopolis (metropolis) in 1957, which was first close to the concept of urban agglomeration. Metropolis refers to the large multi-center urbanization system, which has high continuity and strong internal correlation. This new urban group concept has been accepted by geographical scholars and urban planning practitioners as the origin of urban agglomeration phenomenon research. Zhaolin Gu (2010), a domestic scholar, pointed out that urban agglomeration refers to the aggregate of multiple cities formed by radiating from the central city to the surrounding. Taking urban agglomeration as the main part to promote China's urbanization process has become an important choice of urbanization road with Chinese characteristics. Current academic circles on the concept of urban agglomeration and urban agglomeration is the highest spatial organization of urban development to the mature form, is refers to in a specific region within the scope of general with more than one super-large city as the core, composed of at least three cities for unit, relying on advanced communication infrastructure such as network traffic space which is formed by the compact organization, economic ties, and ultimately achieve highly city and high integration of city groups.

\subsection{Formation Conditions and Driving Forces of Urban Agglomerations}

The emergence of urban agglomerations is a historical process. The center of urban agglomeration concentrates a large number of industries and population through polarization effect, so as to achieve rapid development. With the continuous expansion of the scale of central cities, their strength has been enhanced. Large cities will radiate and drive the surrounding small and medium-sized cities and villages, forming an urban circle. At the same time, with the continuous expansion of the city scale and the continuous improvement and progress of the traffic conditions between cities, the area radiated by neighboring cities is shrinking and regional overlap, and the economic ties between cities are getting closer and the mutual influence is getting bigger, which can be considered as forming an urban cluster. Urban agglomeration is not isolated, therefore, is a closed system, it is with neighboring areas, especially in economically developed town concentration areas within the scope of a particular area closely related to different scale of city (town), relying on the superior natural environment basis and the developed transportation system and information network formed a relatively complete organic whole. Under the new situation of global economic integration and new-type urbanization, every city in the urban agglomeration area plays a special role in regional economic growth and urban function, and plays a powerful radiation role (including agglomeration and evacuation) relying on the urban-rural integration area. The main functions of urban agglomerations are as follows: (1) urban agglomerations can optimize the allocation of resources in a larger scope; (2) urban agglomeration is the main carrier of advanced productive forces; (3) urban agglomerations have a strong radiative driving effect.

\subsection{Research Status and Overview of Urban Agglomerations at Home and Abroad}

\subsubsection{Foreign Studies on Urban Agglomerations}

Foreign scholars have paid attention to the research of urbanized areas for a long time. Foreign scholars mainly focused on the study of metropolitan areas, metropolitan areas (represented by French scholar Gottman) and urban fields (represented by American scholar Friedman) during the period of industrialization. the rapid development and growth of big cities in developing countries led to the research of some scholars, mainly including the urban-rural mixed zone research (Canadian scholar McGee used the urban and rural transition zone to describe the new urbanized areas in developing countries in Asia) and the extended metropolitan area research after 1970.

\subsubsection{Domestic Studies on Cities}

The research on China's urban agglomeration mainly focuses on the research on urbanization system, urban economic region and urban-rural integration. From the perspective of urbanization, Heji Dai(1998) selected 125 basic regional units of structural urban agglomerations from 209 
prefecture-level and above urban political districts by quantitative method, defined 17 urban agglomerations of different scales, and analyzed the distribution status and development trend of China's urban agglomerations. Qian Zhang, Yunfeng Hu, Jiyuan Liu and other scholars (2011) proposed that urban agglomeration is the natural product of a country's industrialization and urbanization to a higher stage, and a new regional unit for the country to participate in global competition and international division of labor. In recent years, the literature on urban agglomeration development by domestic scholars has increased greatly.

\subsubsection{Domestic and International Studies on Urban Agglomerations}

The concept of urban agglomeration is of Chinese characteristics, but the study of urban areas by foreign scholars has sorted out effective theoretical knowledge system for the study of urban agglomeration in China. The research of domestic scholars on urban agglomeration involves a wide range of aspects, including the research on the conceptual level, as well as the research on the formation mechanism, dynamic mechanism and development path of urban agglomeration.

\subsection{Practical Development of Urban Agglomerations}

\subsubsection{World-Class Urban Agglomerations}

At present, there are five worldwide recognized large-scale world-class urban agglomerations, namely, the North Atlantic Coast Urban agglomeration in the northeastern United States, the Great Lakes Urban agglomeration in North America, the Pacific Coast Urban agglomeration in Japan, the British Urban agglomeration, and the Northwest European Urban agglomeration. The World Bank released a report; the Pearl River Delta urban agglomeration overtook Tokyo, Japan, to become the world's most populous and largest urban agglomeration on January $26^{\text {th }}, 2015$.

\subsubsection{China's National Urban Agglomerations}

China's economy is in a period of rapid development, and the development of urban agglomerations has become a new feature of the Era. China has formed the Yangtze river delta urban agglomeration and urban agglomeration in the pearl river delta, Beijing-Tianjin-Hebei urban agglomeration, Zhongyuan urban agglomeration and urban agglomeration in the middle reach of Yangtze river, chongqing urban agglomeration and urban agglomeration, LiaoZhong South Urban Agglomeration and Urban Agglomeration, Shandong Peninsula Urban Agglomeration, the channel west bank beibu gulf urban agglomeration, urban agglomerations in guanzhong plain, call Hubei elm city national urban agglomeration, etc by the end of March 2017. As of March 5, 2018, the state council has approved the total 9 national urban agglomeration.

\section{The Relationship between Rural Revitalization and Urban Agglomeration Development}

\subsection{Relationship between Rural Revitalization and Urbanization}

China is in the stage of urbanization development, and the current urbanization rate is $57.4 \%$. To improve the urbanization rate, we need to narrow the urban-rural gap, improve agricultural production efficiency, increase farmers' income, promote the transfer of rural population to urban areas and increase farmers' non-agricultural income. Urbanization plays an important role in improving farmers' income and labor productivity. Rural revitalization and urbanization should play a synergistic and mutually beneficial role in realizing the integrated development of urban and rural areas.

\subsection{Relationship between Rural Revitalization and Urban Agglomeration Development}

The connotation of rural revitalization is very rich, reflected in economy, culture, civilization and other aspects. The development of urban agglomeration has attracted a large number of farmers to enter the city, which has broadened the farmers' horizon and improved their acceptance of modern culture. When farmers return to their hometowns to revitalize rural development, it will help improve the cultural level of rural areas and enhance the degree of modernization civilization of 
rural areas.

\section{Functions and Roles of Rural Revitalization in Urban Agglomeration Development}

\subsection{The First Function of Rural Revitalization: To Provide New Growth Impetus for Urban Agglomeration Economy}

Country as city feedback as well as a principal part of the counter urbanization, rural revitalization of the first function is to provide a new round of economic growth for regional urban agglomeration, the new rural economy and infrastructure construction lags behind that of the city, there is no economic products and capital, it is only through the feedback of the infrastructure construction, urban agglomerations of various provinces and cities in the near future cooperation set up by national or similar linkage assistance "Zhejiang Businessman Village", to make rural on thought into the thought of "Win-win Cooperation".

\subsection{The Second Function of Rural Revitalization: To Implant "Rural" Excellent Culture in the Development of Urban Agglomerations}

As another turning point of economic growth, the second function of rural revitalization lies in its demonstration of the "spirit of contract" of urban agglomeration. At present, due to the fast pace of life, the city has formed an atmosphere of advocating interests. "Rural revitalization" to urban agglomeration reaction in cultural aspects, through the establishment of "township spirit", honest, simple and honest, steadfast, the spirit of cooperation makes the rural cooperatives and so on a series of farmer's cooperative development, it also provides many ideas for many of the urban agglomeration, since there are two province or more linkage support the countryside, there will be many provinces of mutual support for poverty-stricken counties. Trade in agricultural products or the products of major urban enterprises. Rural revitalization forms "residents are attached to their towns because they are strong and independent; they care about their towns because they participate in their management; they love their towns because they cannot help but cherish their fate.

\subsection{The Third Function of Rural Revitalization: To Provide Urban Agglomeration with Basic Tertiary Industry Income Increment}

This paper argues that the third function of rural revitalization in urban agglomeration is to "provide basic tertiary industry income increment for urban agglomeration". At present, more urban agglomeration in the development of tourism is mostly to build humanistic landscape. Compared with rural natural landscape, humanistic landscape is not as magnificent and magnificent as natural landscape. The value-added of the tertiary industry can be fully carried out from the rural unique natural landscape and landform, sharing the pressure of urban agglomeration population. On the other hand, rural revitalization is also to redistribute and sell the surplus of products in urban agglomerations. There is a large market in rural areas, which can help the new science and technology industries in urban agglomerations to conduct product demonstration and research as much as possible.

\subsection{Fourth Function of Rural Revitalization: To Provide Talent Support for Urban Agglomeration Construction}

With the in-depth strategic deployment of rural revitalization, the quality of rural labor force will be continuously improved, and the production efficiency of rural agriculture will be greatly improved. More surplus labor force will be transferred to urban agglomerations, providing talent support for urban agglomeration construction.

\subsection{The Role of Rural Revitalization: The Role of Nanny and Brother}

Rural revitalization plays a role that I personally think belongs to "nanny and brother". The role of the nanny is that the countryside will become the next growth power source for the economic growth of the urban agglomeration and maintain the development of the urban agglomeration. It is also an opportunity for the collaborative development and win-win cooperation between the rural 
and urban areas. The so-called brothers' role, rural revitalization is bound to threaten the status of the city. This case is similar to the case of shiyan city in Hubei Province in the last century. Shiyan City began to be the jurisdiction town of Yunyang City and became the provincial prefecture of Yunyang City after more than ten years of development. Such examples have to think about the country in the revitalization of the position, is bound to make the development of urban agglomeration threatened by rural revitalization, brother role is not only to let the country get more development opportunities in the future, as well as the power, and draw lessons from "the villages and towns spirit" thinking of the future "the villages and towns and contract spirit," to make the coordinated development of urban and rural.

\section{Conclusion}

To sum up, with the deepening of the strategy of rejuvenating rural deployment, will form a series of policies and system, to realize rural economic development, improve personnel quality, appeal to the people, the resources of urban agglomerations function, makes the country revitalization strategy in the urban agglomerations function and role in the development of more outstanding, to make full use of the linkage between urban and rural development, interaction, and promote rural revitalization of the realization of the strategic objectives and tasks, but also can realize urban agglomeration development back-feeding rural development, to bring a good development for rural development, to realize the harmonious development of rural and urban agglomerations.

\section{References}

[1] Xin Zhang. On Rural Revitalization and Regional Coordinated Development Driven by Urbanization [J]. Environmental Economy, 2018(z1):70-73

[2] Yanbing Chen. On the Implementation of Rural Revitalization Strategy to Promote Urban-rural Integration Development -- and the Experience of Learning from the 19th CPC Report [J]. China Economic and Trade Guide, 2017(34):52-55.

[3] Zhenwei Peng. On the Development of Small Towns and Implementation of Rural Revitalization Strategy [J]. Urban and Rural Planning, 2018(1):11-16

[4] Guoxiang Li. On Several Important Relationships Correctly Recognized and Dealt with to Achieve Rural Industrial Prosperity [J]. Zhongzhou Journal, 2018(1):32-38. 